\title{
Google Image Swirl: A Large-Scale Content-Based Image Browsing System
}

\author{
Yushi Jing, Henry A. Rowley, Charles Rosenberg, Jingbin Wang, \\ Marius Pasca, Yi Liu, Ming Zhao, Michele Covell \\ $\{j$ ing, har, chuck, jingbinw, mars, yliu, mingzhao, covell\}@google.com \\ Google Inc., Mountain View, California, United States of America
}

\begin{abstract}
We demonstrate the first large-scale image browsing system applied to 200,000 popular queries which utilizes image content to organize image search results. Given a query, the system extracts image content features such as color, shape, local features, face signatures and metadata from up to 1000 image results, and hierarchically clusters them to form an exemplar tree. A dynamic web-based user interface allows the user to navigate this hierarchy, allowing fast and interactive browsing. The exemplars of each cluster provide a comprehensive visual overview of the query results, and allow the user to quickly navigate to the images of interest.
\end{abstract}

\section{Exemplar Tree}

This work organizes image search results into an exemplar tree. We begin organizing up to 1000 results of an image search query by building a pairwise similarity matrix among these images. The similarity computation is based on a combination of image features such as color, texture, local features, face signatures, and the metadata associated with the images. We then perform clustering to partition the search results into hierarchical clusters, each associated with a representative, or exemplar, image. The hierarchical clusters for each query are pre-computed for this demonstration. For more detail, see [3].

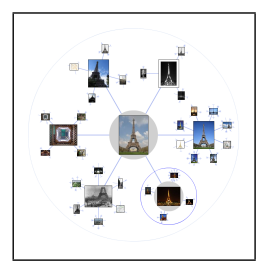

Figure 1. Radial layout of cluster hierarchy.

\section{User Interface}

After hierarchical clustering has been performed, the results of an image search query are organized in the structure of a tree. A number of options exist for how to present such a tree to the user. Beyond the typical layered diagram

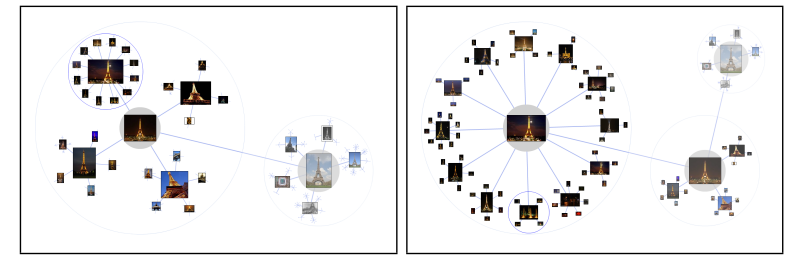

Figure 2. Expanding sub-clusters selected by user.

used to illustrate tree data data structures, there are many options in the literature, including hyperbolic geometry to better utilize space [4], and a variety of approaches based on tree-maps [1]. In this demonstration, we used radial layout in which each layer of the tree is arranged radially around its parent, see Figure 1. When the user selects a branch of the tree to explore, it is separated from the parent and expanded, while the parent is shrunk to make space, see Figure 2. The rearrangement is animated to allow the user to follow the change without getting lost.

\section{Future Work}

We hope to expand this demonstration to provide the users with text annotations on the clusters, to allow browsing to related queries and to incorporate other media types. We also hope to incorporate a semantic ontology such as WordNet [2] into the generation of the exemplar tree.

\section{References}

[1] B. Bederson, B. Shneiderman, and M. Wattenberg. Ordered and quantum treemaps: Making effective use of 2D space to display hierarchies. ACM Transactions on Graphics, 21(4):833-854, 2002.

[2] C. Fellbaum, editor. WordNet: An electronic lexical database. MIT Press, 1998.

[3] Y. Jing, H. A. Rowley, C. Rosenberg, J. Wang, and M. Covell. Visualizing web images via google image swirl. In NIPS Workshop on Statistical Machine Learning for Visual Analytics, 2009.

[4] J. Lamping and R. Rao. Laying out and visualizing large trees using a hyperbolic space. In Proceedings of the 7th annual ACM symposium on User interface software and technology, pages 13-14. ACM New York, NY, USA, 1994. 